\title{
NSTX Filament Preionization And Glow Discharge Cleaning Systems
}

\author{
H. W. Kugel, W. Blanchard, G. D'Amico, R. Gernhardt, T. Provost \\ Princeton Plasma Physics Laboratory, Princeton University, Princeton NJ 08543-0451 USA
}

\begin{abstract}
Initial NSTX GDC experiments were performed with one moveable anode and a biased filament preionization system that allowed $\mathrm{D}_{2}$ and He Glow Discharge breakdowns at the actual operating pressure, voltage and current. The biased filament system was also operated continuously during ohmic operations, and used to reduce volt-sec consumption for February 1999 plasma discharges up to 280 KA. An upgraded system has been installed with 2 fixed wall anodes and 3 biased filaments; 2 on the mid-plane and one in the divertor region; all separately controllable remotely using a PLC system. Recent applications include assisting in preionization for $800 \mathrm{KA}$ plasma discharges.
\end{abstract}

\section{INTRODUCTION}

The National Spherical Torus Experiment (NSTX) is designed to investigate the physics of Spherical Tori (ST) in a device that can produce non-inductively sustained high- $\beta$ discharges in the $1 \mathrm{MA}$ regime and to explore approaches toward a small, economic, high power density Volume Neutron Source (VNS) and an ST reactor core.[1]

Initial ohmic operations began in February 1999 with the vessel configured with only the Center Column ("Center Stack").[2] The Center Stack clad with graphite tiles, a small outboard graphite limiter, and 4 flux loops were the only hardware in the otherwise empty SS vessel. In preparation for this work, since the vacuum vessel bakeout capability was not scheduled to be available until later in CY1999, the internal surfaces of the vacuum vessel were conditioned by applying $\mathrm{D}_{2}$ Glow Discharge Cleaning $\left(\mathrm{D}_{2} \mathrm{GDC}\right)$ for 39 hours followed by 4 hours of HeGDC. Subsequently, $280 \mathrm{KA}$ plasma discharges were readily obtained using a preliminary Filament Preionization System. After the brief operation in February 1999, the vessel was vented for additional upgrades. During the vent, considerable internal hardware, e.g., the divertor, passive plates, graphite tiles, and in-vessel sensors [2] were installed. The vessel was evacuated in August 1999, and in preparation for plasma operations, the vacuum was prepared by the application of about 140 hours of $\mathrm{D}_{2} \mathrm{GDC}$ and 20 hours of HeGDC using a double fixed anode GDC system with preionization filaments that allow GDC initiation at the operating pressure and voltage. Plasma operations resumed in September 1999, and plasma discharges of over $800 \mathrm{KA}$ were readily achieved. Plasma breakdown was assisted using the upgraded Filament Preionization System described below together with an Electron Cyclotron Preionization System that will be used for Coaxial Helicity Injection (CHI) experiments.

\section{Experimental Geometry for Initial Tests}

A common method for initiating $\mathrm{D}_{2}$ and $\mathrm{He}$ GDC is to start with fill pressure in the range of 20 to 40 mTorr with an applied bias in the range of 600 to $800 \mathrm{~V}$. Once breakdown is achieved and GDC is initiated, the pressure is quickly reduced to a few mTorr, or lower, and the applied bias to about 250 to $600 \mathrm{~V}$. This is done to reduce violent arcing and sputtering events, and to reduce the load on the torus vacuum pumping system which is often kept in the normal high vacuum mode during GDC. Hence, the capability to

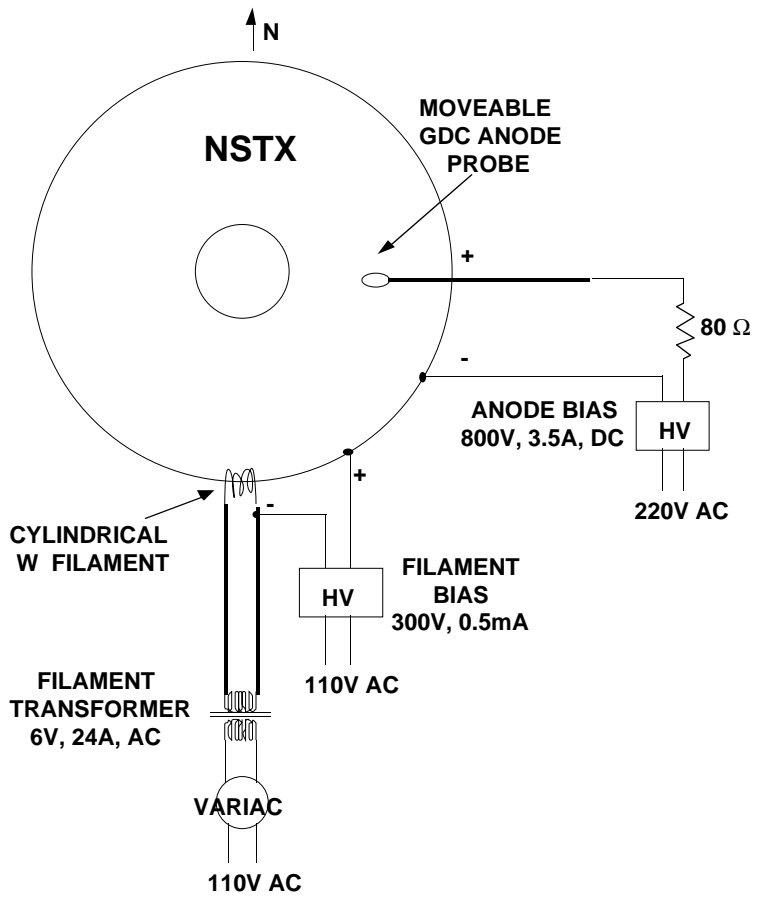

Fig. 1. Partial schematic diagram of initial configuration used for GDC experiments and cleanup for February 1999 ohmic plasma operations. In addition, the filament was used to provide preionization for initiation of February 1999 plasma discharges. 
initiate GDC breakdown at the actual operating pressure and voltage, provides the advantages of minimizing coatings from arcing and sputtering, minimizes stress to the pumping system, and greatly simplifies the controls needed for remote operation and to perform automated GDC between plasma discharges.

Prior to February 1999 plasma operations, with the vacuum vessel in the initial relatively "empty" configuration, a series of experiments were performed to investigate $D_{2}$ and He GDC characteristics in NSTX. Fig.1 shows the experimental geometry. A variable position anode probe was used which consisted of one 304-SS blade, $13.65 \mathrm{~cm} \mathrm{x}$ $5.08 \mathrm{~cm} \times 0.16 \mathrm{~cm}$. The applied bias directed ions to the vessel wall. A biased filament was positioned about $90^{\circ}$ toroidally from the moveable anode. The filament consisted of a 10 turn coil of $0.102 \mathrm{~cm}$ diameter tungsten wire oriented with its long axis parallel to the TF field. The filament could be biased so as to direct the emitted electrons to the walls of the vessel.

\section{Results of Initial GDC Experiments}

\section{A. GDC Range With Zero Filament Bias}

The first experiments were performed with zero filament bias and the anode positioned about midway between the Center Stack and the outer wall of the vessel $(68.90 \mathrm{~cm}$ from the wall). Applying a no-load anode bias of about 800 $\mathrm{V}$, the $\mathrm{D}_{2} \mathrm{GDC}$ pressure threshold was about 25-30 mTorr. After initiation of $\mathrm{D}_{2} \mathrm{GDC}$, the full load bias dropped into the range from $300 \mathrm{~V}, 1.8 \mathrm{~A}$ to $500 \mathrm{~V}, 2.5 \mathrm{~A}$, a variation that was not investigated although there were indications that this threshold depended on vacuum conditions and the operating history of the probe. In the case of $\mathrm{He}$, with a noload anode bias of about $800 \mathrm{~V}$, the HeGDC pressure threshold was about 10-15 mTorr. After initiation of the HeGDC, the full load bias dropped to about $450 \mathrm{~V}$ with currents of $3.5 \mathrm{~A}$.

It was found that the $\mathrm{D}_{2}$ GDC breakdown pressure threshold could be reduced by establishing a suitable pressure, and then applying the anode bias in an abrupt step rather than applying it at a constant level while the pressure was raised. This effect may be due to power supply turn-on transients. Under these conditions, for example, for a preset operating voltage of $500 \mathrm{~V}$, the $\mathrm{D}_{2} \mathrm{GDC}$ breakdown pressure was reduced from $25-30 \mathrm{mT}$ to about 15 mTorr. The lower limit for stable operation of both $\mathrm{D}_{2} \mathrm{GDC}$ and HeGDC was found to be about $2 \mathrm{mT}$. Operation at about $1 \mathrm{mT}$ was intermittent and was not pursued.

\section{B. Threshold Experiments, With Filament Bias}

Filaments currents up to 30 A were applied with and without filament bias. It was found that an unbiased filament did not reduce the breakdown thresholds or improve stabiliy near the low pressure limit. However, with applied bias from 8 to $300 \mathrm{~V}$, and emission currents from 0.1 to $0.9 \mathrm{~mA}$, corresponding to filament AC currents of 23 to $25 \mathrm{~A}$, it was found that both $\mathrm{D}_{2} \mathrm{GDC}$ and HeGDC could be initiated reliably at pressures of $2 \mathrm{mT}$. At $1 \mathrm{mT}$, the behavior was intermittent and similar to the no-bias case described above (II. A).

After the above measurements, the moveable anode probe was withdrawn from its position about midway between the Center Stack and the outer wall to a distance $30.80 \mathrm{~cm}$ from the outer wall. The above $\mathrm{D}_{2}$ and $\mathrm{He}$ GDC initiation experiments were repeated with filament biases in the range described above, and it was found that the above results could be obtained with the anode closer to the outer wall. These results provided the database for the design of a double fixed probe GDC system capable of reliably striking reproducible discharges at 2-4 $\mathrm{mT}$ which could serve as the basis for automating HeGDC between plasma discharges.

\section{Cleanup With Initial Configuration}

After the above experiments, the moveable anode probe was returned to its initial midway position, and extensive GDC cleanup of the vessel was started. Fig.2 shows selected partial pressures measured with a residual Gas Analyzer during the $\mathrm{D}_{2} \mathrm{GDC}$ and HeGDC. Due to both physical and chemical sputtering, the desorption of the hydrogen and deuterium water molecule complexes at ( $\mathrm{A}=17-20)$, and $\mathrm{CO}$ (and other $\mathrm{A}=28$ masses) increased significantly during $\mathrm{D}_{2} \mathrm{GDC}$, thereby accelerating their removal from the vessel via the Torus Vacuum Pumping System which was fully open during this operation. HeGDC was applied following $\mathrm{D}_{2} \mathrm{GDC}$ to remove the residual $\mathrm{D}_{2}$ and hydrocarbons.

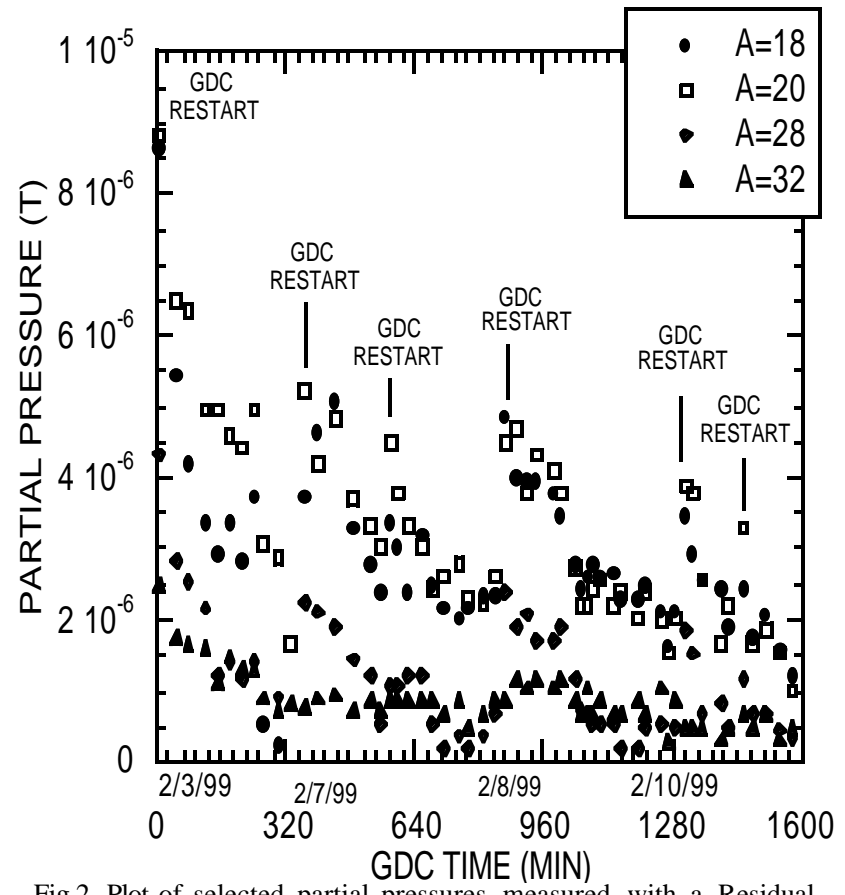

Fig.2. Plot of selected partial pressures measured with a Residual Gas Analyzer during $D_{2}$ GDC with Feb. 1999 configuration. 


\section{Preionization of Initial ohmic Discharges}

During the initial February 1999 NSTX startup, after repeated discharge attempts, which included experimental difficulties with an incomplete field null and other field asymmetries, it was found that the first ohmic discharge was limited to a plasma current of about 20KA. The application of filament preionization using the system described above with a 24 A filament AC current with a 300 V. bias and about $0.4 \mathrm{~A}$ of emission current had an immediate effect on improving the discharge startup characteristics. Subsequently, NSTX ohmic discharges were able to increase with each successive discharge to plasma currents of about 280 KA (see Fig.3).

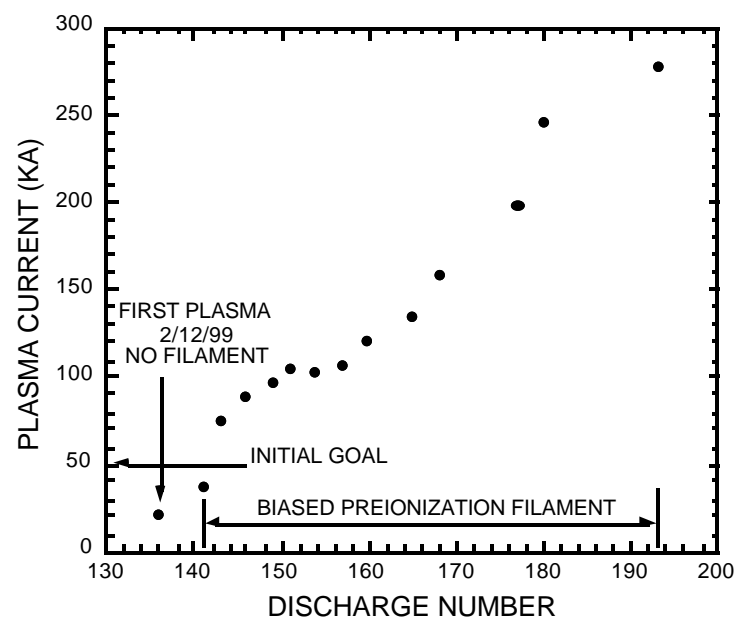

Fig.3. Plot of February 1999 Plasma Current versus Discharge Number. Indicated is the first plasma discharge which was limited to about $20 \mathrm{KA}$ without filament preionization. The startup of the subsequent discharges was facilitated with biased Filament Preionization.

\section{Geometry of Present Configuration}

Fig.4 shows the toroidal locations of the present double fixed GDC probes and the 3 sets of the Preionization Filaments. The fixed GDC probes are located near the midplane. Fig.5 shows a partial schematic of the Bay-G GDC anode. The GDC anodes are $25.4 \mathrm{~cm}$ long and located about $3 \mathrm{~cm}$ (Bay-G) and $13 \mathrm{~cm}$ (Bay-K), behind the outermost RF antenna limiter, respectively. Two of the three filaments are located near the midplane at the same Bay port flanges as the midplane GDC anodes. The third filament is mounted in the Bay-D lower divertor port which is close to the CHI breakdown region. Fig.6 shows partial schematic diagram of the Bay-G filament holder. Each filament holder [3] contains two filaments, an electrically connected filament and a spare. The Bay-G filament is about $6 \mathrm{~cm}$ from the RF Limiter and the Bay-K filament is about $16 \mathrm{~cm}$ form the RF limiter. The Bay-D divertor -fila-

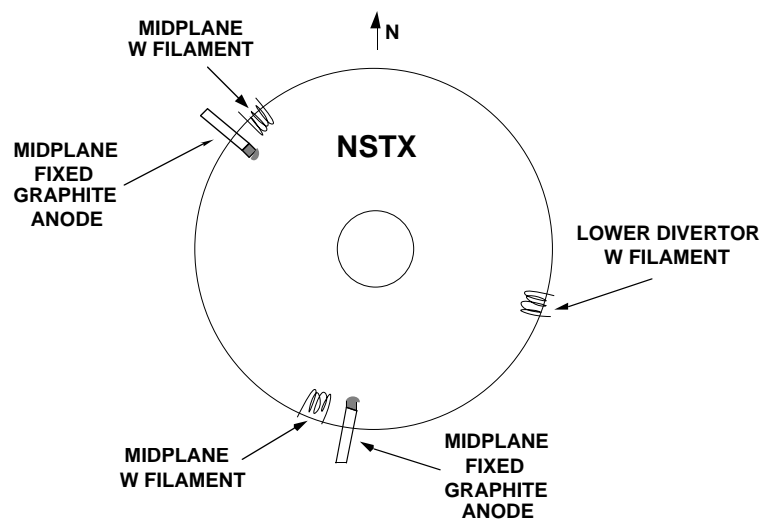

Fig.4. A partial schematic drawing of the present Filament Preionization system and the Fixed Probe GDC system.

-ment is a few cm below the plane of the Outer Divertor and has a line-of-sight to the gap between the inner and outer divertors, across which will be applied the $\mathrm{CHI}$ breakdown voltage. The filaments are fabricated from $0.0 .051 \mathrm{~cm}$ dia. $\mathrm{W}$ wire in a $2.5 \mathrm{~cm}$ long cylindrical form of 10 turns. The long axis of then cylindrical coils are oriented parallel to the NSTX TF field.

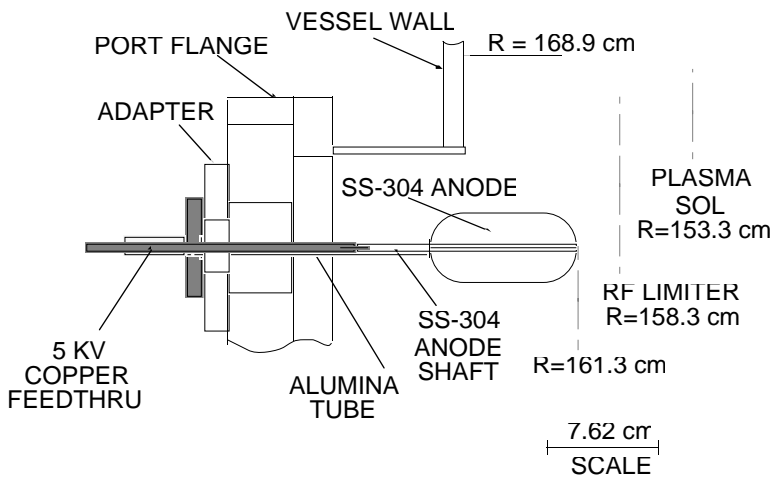

Fig.5. Partial schematic of the Bay-G anode mounted on a $5 \mathrm{KV}$ feedthrough [3] located about $3 \mathrm{~cm}$ behind the outermost RF antenna limiter

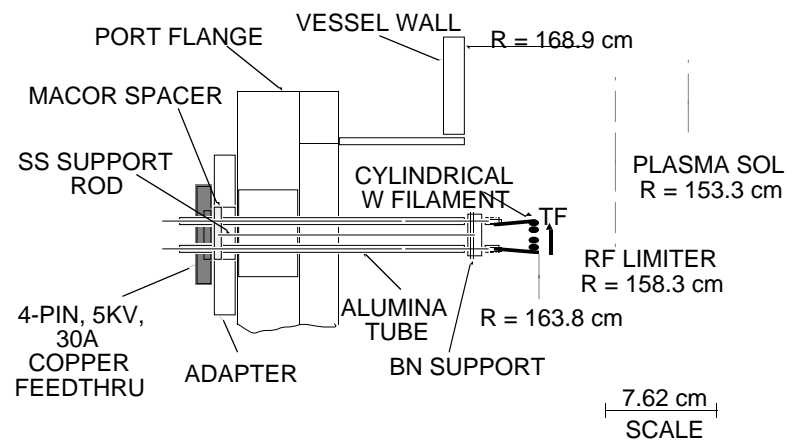

Fig.6. Partial schematic diagram of the Bay-G filament holder (4pin copper, $5 \mathrm{KV}$ feedthrough [3]) which holds two filaments. One filament is electrically connected and the other is a spare. 


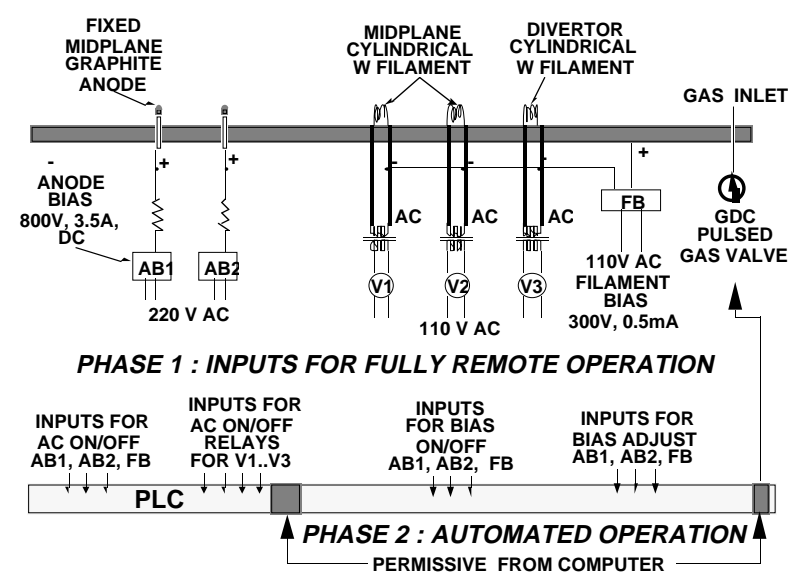

Fig.7. Partial schematic elementary electrical diagram of PLC Controls for Preionization Filament and GDC system.

\section{PLC Control System}

Fig.7 shows an elementary electrical schematic diagram of the Filament Preionization and Fixed Probe GDC systems connected to a Programmable Logic Controller (PLC). The system is presently configured for remote manual control. Eventually, it will be automated so that upon a computer generated permissive signal, the PLC will send signals resulting in the closing of the diagnostic window shutters and torus interface gate valves, the filling of the torus with He GDC gas, the starting of the Preionization Filaments, and the application of GDC anode bias. The HeGDC will continue for a few minutes duration between NSTX plasma discharges to maintain wall conditioning. After the HeGDC is completed, the PLC will initiate and the reverse process to reestablish the readiness of the vessel for the next discharge. The present PLC software has been configured to allow for this expansion from manual to automated operation.

\section{Results of Present Filament Preionization and GDC Experiments}

The upgraded Preionization Filament system is capable of about a factor of 10 more emission current. Fig. 8 shows a comparison of the emission current obtained with the February 1999 prototype configuration and the upgraded configuration. The scatter in the data for the February 1999 configuration was due to having been measured at different times with slightly different filament currents. In the case of the upgraded system, it is seen that considerable emission is obtained at relatively small bias voltages. The experimental program has not yet investigated the minimum required bias voltage for either GDC initiation or plasma startup. Presently, about $400 \mathrm{~V}$ filament bias is applied for both functions. The bay- $\mathrm{G}$ and Bay-K filaments on the midplane have been operated continuously during plasma discharges in TF fields up $0.3 \mathrm{~T}$ and plasma discharges up $800 \mathrm{KA}$ to date.

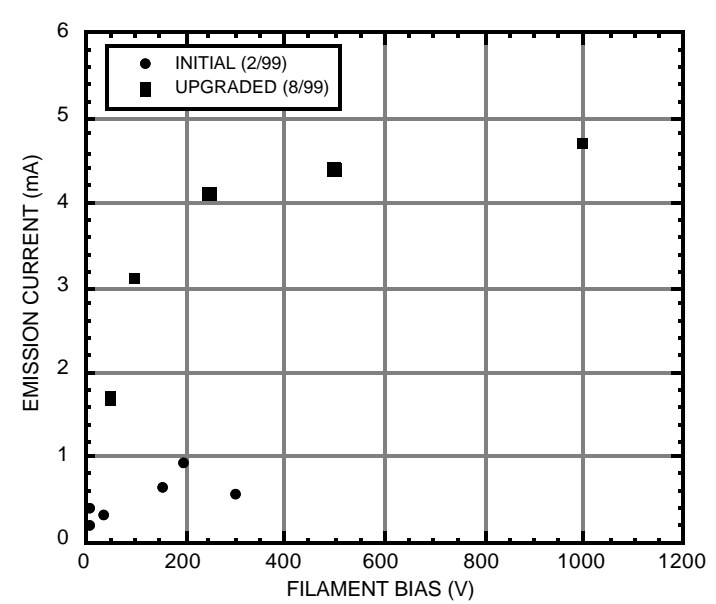

Fig.8. Plot of Bay-K biased filament current versus applied bias voltage for initial February 199 configuration, and the upgraded configuration (refer to text).

Due to the extensive construction activity within the vessel during the March-July 1999 vent, the large amount of graphite added, and the absence of a bakeout system, the preparation for plasma operations included the application of about 140 hours of $\mathrm{D}_{2}$ GDC and 20 hours of HeGDC using the double fixed anode GDC system with preionization filaments. Most of this GDC was performed with the vessel at room temperature. About 10 hours of $\mathrm{D}_{2} \mathrm{GDC}$ was performed during the initial vessel bakeout with the Center Stack at about $220{ }^{\circ} \mathrm{C}$ and the passive plates about $60^{\circ} \mathrm{C}$. Due to both physical and chemical sputtering, the desorption of the hydrogen and deuterium water molecule complexes at $(\mathrm{A}=17-20)$ and $\mathrm{CO}$ (and other $\mathrm{A}=28$ masses) increased significantly during $\mathrm{D}_{2} \mathrm{GDC}$, thereby accelerating their removal from the vessel via the Torus Vacuum Pumping System which was fully open during this operation. HeGDC was applied following $\mathrm{D}_{2} \mathrm{GDC}$ to remove the residual $\mathrm{D}_{2}$ and hydrocarbons. This resulted in vessel base pressures of $6.7 \times 10^{-8}$ Torr and plasma conditions thtat allowed the relatively prompt achievement of $800 \mathrm{KA}$ plasma discharges.

\section{Acknowledgments}

The authors would like to acknowledge the technical contributions of M. Anderson, J. Bartzak, W. Derry, S. Edwards, G. Gibilisco, K. Gilton, S. Jurcznski, P. Kivler, R. Knols, G. Rossi, F. Simmons Jr., S. Vinson, and J. Winston. Work Supported by USDOE Contract No. DE-AC02-76-CHO3073

\section{References}

1. S. M. Kaye et al., "Physics Design of the national Spherical Torus Experiment", Fus. Technol. 36(1), 16 (1999).

2. H. W. Kugel et al., "NSTX High Temperature Sensor Systems", in these Proceedings.

3.Insulator Seal, Inc., 23874-B Cabot Blvd., Hayward, CA 945451661, USA. 